\title{
UNA NUEVA ESPECIE Y UN NUEVO REGISTRO DE DRYMONIA (GESNERIACEAE) EN COSTA RICA
}

\author{
RicARDO KRIEBEL \\ Botany Dept., California Academy of Sciences, 875 Howard Street, San Francisco, CA, 94103, USA \\ rkriebel@inbio.ac.cr
}

\begin{abstract}
Drymonia glandulosa Kriebel, a new species restricted to the northern Caribbean lowlands of Costa Rica is described and compared to D. conchocalyx. Drymonia mortoniana Wiehler is recognized as a distinct species and its presence in Costa Rica is a new record for the country's flora.
\end{abstract}

Resumen. Se describe Drymonia glandulosa Kriebel, una nueva especie que habita en las bajuras del norte de la vertiente caribe de Costa Rica, y se compara con D. conchocalyx. Drymonia mortoniana Wiehler se reconoce como especie válida y representa un nuevo registro en la flora del país.

Palabras Clave / Key Words: Gesneriaceae, Drymonia conchocalyx, Drymonia glandulosa, Drymonia mortoniana, Costa Rica

La exploración botánica no había abarcado los bosques muy húmedos de la zona norte de Costa Rica hasta fechas recientes. Fruto de esta exploración, son varios registros nuevos de plantas en el país; por ejemplo, Clidemia involucrata DC. (Melastomataceae), Pouteria cuspidata (A.DC.) Baehni (Sapotaceae) y Psychotria zevallosii C.M.Taylor (Rubiaceae), así como varias especies nuevas: Miconia sp. nova, Symplocos naniflora L.Kelly \& Almeda, Symplocos striata Kriebel \& N.Zamora y Faramea zamorae A.Rodríguez. En este artículo se describe otra especie nueva, Drymonia glandulosa Kriebel, proveniente de la región de Boca Tapada en San Carlos, Costa Rica, de la cual se conocen unas pocas plantas vivas y posiblemente se encuentra en peligro de extinción en pequeños bosques remanentes en la zona.

El género Drymonia Mart. es el segundo más grande de la familia Gesneriaceae en Costa Rica, con cerca de 22 especies, y 140 especies en los bosques neotropicales (Burtt \& Wiehler 1995). En Gesneriaceae de Costa Rica, solamente el género Columnea, con 36 especies, es más grande que Drymonia. De las 22 especies costarricenses de Drymonia, el 50\% (11 spp.) se encuentran restringidas en distribución desde Nicaragua hasta Panamá, cuatro hasta ahora son endémicas en Costa Rica [ $D$. fimbriata C.V.Morton, $D$. glandulosa Kriebel, D. peltata (Oliv.) H.E.Moore y D. $s p$. nova] y cuatro se comparten solamente con Panamá (D. folsomii L.E.Skog, D. mortoniana Wiehler, D. parviflora Hanst. y D. rubra C.V.Morton).

Algunas características del género Drymonia incluyen su hábito frecuente de bejuco, que es casi único entre las Gesneriáceas de Costa Rica. Otras características son las flores axilares, brácteas en ocasiones llamativas, cáliz y corola de variados colores, la última usualmente en forma de embudo o trompeta, con un espolón evidente en la base y lóbulos de margen aserrado a fimbriado, anteras dehiscentes por poros y frutos por lo general cápsulas bivalvadas (Wiehler 1983). El carácter de dehiscencia poricida de las anteras es uno de los más distintivos del género. En Gesneriaceae, el único otro género con poros es Codonanthe. Los poros en las anteras de Drymonia son basales, en Codonanthe apicales (Wiehler 1983).

\section{Drymonia glandulosa Kriebel, sp. nova}

TIPO: Costa Rica. Alajuela: San Carlos, 7 km NE de Boca Tapada, Laguna del Lagarto Lodge, $10^{\circ} 41^{\prime} 10^{\prime \prime} \mathrm{N}$, $84^{\circ} 10^{\prime} 50$ 'W, 90 m, 22-28 febr 2004, R. Kriebel 4414 (Holotipo: INB, dos láminas). Figs. 1 y 2.

A Drymonia conchocalyci Hanst. affinis, sed petiolo lepidoto, pedicelo minore, corola violacea cum superficie interna glandulosa, stylo minore glandulosopilosoque differt.

Arbustos epífitos con ramas escandentes en el sotobosque de bosques poco alterados, con tallos esencialmente glabros, más o menos teretes, verdes tornándose morados, con raíces adventicias; entrenudos 3-10.5 $\mathrm{cm}$ de largo y 3-6 $\mathrm{mm}$ de diámetro. Hojas de cada nudo generalmente del mismo tamaño, pecioladas; pecíolos (2.5-)3.5-6 cm de largo y 1$3.25 \mathrm{~mm}$ de diámetro, escamosos, verdes a morados; láminas 9.5-23 x 5.2-11 cm, elíptico-ovadas, glabras 
y verdes en ambas superficies, subcoriáceas, al secar levemente revolutas, el ápice abrupto-acuminado, la base aguda a casi redondeada, el margen entero y con diminutos e inconspicuos dientes aparentemente glandulares, venas 7-9 en cada lado, prominentes en el envés. Inflorescencia de flores axilares solitarias (posiblemente también en pares); pedúnculos ausentes; brácteas inconspicuas; pedicelos ca. 6-13 mm de largo, glabros. Flores con los lóbulos ventrales y laterales del cáliz de mayor tamaño, 1.5-2.5 cm de largo y connados por ca. 0.6-1.2 cm, oblongos a ovados, el ápice agudo a redondeado, el margen serrulado, el lóbulo dorsal y que cubre el espolón de la corola usualmente de menor tamaño, todos verdes, con o sin manchas inconspicuas moradas hacia el ápice de los lóbulos; corola 5.5-7 $\mathrm{cm}$, externamente glabra, el tubo externamente blanco con tonos de morado, los lóbulos y el interior morados con manchas blancas adentro del tubo ventralmente, internamente cubierta con tricomas glandulares especialmente hacia la boca y hacia el espolón; espolón $5 \mathrm{~mm}$ de largo, glabro, la boca bilabiada, lóbulos 5 , glabros, con márgenes erosos, 1.1-1.8 x 1.2-2 cm, los tres ventrales de mayor tamaño, especialmente el central; filamentos ca. $3 \mathrm{~cm}$ de largo, adnados en la base a la corola por 3-4 mm; anteras de $6 \times 1.25-2.5$ $\mathrm{mm}$; disco de una glándula nectarífera dorsal, glabra y de ápice agudo; ovario 5-6 mm de largo, estriguloso; estilo ca. $1.5-3 \mathrm{~cm}$ de largo, densamente glandularpiloso, estigma estomatomorfo. Fruto no observado.

DistRIBUCIÓN, HÁBITAT Y BIOLOGÍA. Drymoniaglandulosa crece en los bosques muy húmedos de la zona norte de Costa Rica, a ca. $90 \mathrm{~m}$ de altitud. La especie es notablemente rara, posiblemente por su preferencia por bosques poco alterados, los cuales son escasos en la zona. Algunas de las especies de árboles y arbustos más comunes en estos bosques son Pentaclethra macroloba (Willd.) Kuntze (Fabaceae/Mim.), Ferdinandusa panamensis Standl. \& L.O.Williams (Rubiaceae), Rinorea deflexiflora Bartlett (Violaceae), Eschweilera costaricensis S.A.Mori y Lecythis ampla Miers (ambas de Lecythidaceae), Ryania speciosa Vahl (Flacourtiaceae), Couma macrocarpa Barb. Rodr. (Apocynaceae), Henriettea odorata (Markgr.) Almeda, Miconia gracilis Triana, M. elata (Sw.) DC. y M. lateriflora Cogn. (las cuatro de Melastomataceae), Qualea polychroma Stafleu (Vochysiaceae) y Elaeoluma glabrescens (Mart. \& Eichler) Aubrév. (Sapotaceae). Algunas Gesneriáceas simpátricas con D. glandulosa son Besleria columneoides Hanst., Columnea gloriosa Sprague, C. nicaraguensis Oerst., C. purpurata Hanst., Drymonia macrophylla (Oerst.)
H.E.Moore, Drymonia submarginalis Gómez-Laur. \& Chavarría y Paradrymonia decurrens (C.V.Morton) Wiehler. La única corola disectada contenía alrededor de 50 trips (Thysanoptera).

Etimología. El epíteto glandulosa hace alusión al indumento glandular del interior de la corola y del estilo, uno de los caracteres diagnósticos de la nueva especie.

Drymonia glandulosa es muy similar a D. conchocalyx Hanst., ambas comparten láminas foliares glabras, coriáceas, con el margen esencialmente entero, venación terciaria inconspicua, lóbulos ventrales y laterales del cáliz connados en parte de su longitud, corola larga y estigma estomatomorfo. En su interpretación de D. conchocalyx, Skog (1979) incluye a $D$. brevipes Morton, nombre de una entidad descrita de Cerro Pilón, Coclé, Panamá, puesto que las únicas diferencias entre ambas son la unión de los lóbulos del cáliz por mayor longitud y pedicelos más cortos. Aunque este último carácter se utiliza también en la separación de D. conchocalyx y D. glandulosa, otras diferencias se enumeran en la siguente clave para su mejor distinción:

Hojas de cada par principalmente isofilas, elípticoovadas; pecíolos conspicuamente escamosos en plantas vivas y al secar, (2.5-)3.5-6 cm; pedicelos de $0.6-1.3 \mathrm{~cm}$; cáliz esencialmente verde; corola con el tubo externamente blanco con o sin manchas inconspicuas moradas, la boca y el interior principalmente morados, densamente glandular-pilosa en el lado interno; estilo ca. 1.5$3 \mathrm{~cm}$, densamente glandular-piloso; restringida a la zona norte (Boca Tapada), Costa Rica, $90 \mathrm{~m}$ D. glandulosa

Hojas de cada par usualmente anisofilas, ocasionalmente isofilas, elípticas a elípticooblongas; pecíolos en ocasiones escamosos en plantas vivas pero nunca al secar, 1.5-3(-4) cm; pedicelos de (1.2-) 2-4.5 (-8.5) cm; cáliz rosado, excepcionalmente verde; corola principalmente rosada, glabra internamente; estilo ca. $4 \mathrm{~cm}$, glabro; presente en la vertiente caribe de las cordilleras, Costa Rica y Panamá, 700-1900 m

D. conchocalyx

Otro aspecto que llama la atención entre ambas especies es la diferencia en su floración. En Drymonia conchocalyx las plantas comúnmente presentan muchas flores; en cambio, en D. glandulosa ha 
resultado difícil encontrar plantas con flores. Por eso existen pocos especímenes.

Paratipo: Costa Rica. Alajuela: San Carlos, $7 \mathrm{~km}$ NE de Boca Tapada, Laguna del Lagarto Lodge, $10^{\circ} 41^{\prime} 10^{\prime \prime} \mathrm{N}, 84^{\circ} 10^{\prime} 50^{\prime \prime} \mathrm{W}, 90 \mathrm{~m}, 6$ dic 2004 (fl.), $R$. Kriebel 4974 (INB).

A continuación se registra por primera vez en Costa Rica Drymonia mortoniana, una especie rara cuyo nombre anteriormente se ha considerado sinónimo de D. stenophylla (Donn.Sm.) H.E.Moore.

Drymonia mortoniana Wiehler, Phytología 27: 325. 1973, nom. nov. Drymonia parvifolia C.V.Morton, Ann. Missouri Bot. Gard. 29: 56. 1942, nom. illegit., non Drymonia parvifolia Griseb., Fl. Brit. W. I. 463. 1862. Tipo: Panamá; Chiriquí, P.H. Allen 1949 (Holotipo: MO!).

Cuando Conrad V. Morton describió Drymonia parvifolia de Panamá, no se percató de que dicho epíteto ya había sido utilizado por Grisebach en 1862 para una especie colombiana, hoy sinónimo de $D$. serrulata (Jacq.) Mart. (Skog 1979), razón por la cual Wiehler asignó el nombre nuevo D. mortoniana. En Flora of Panama, Skog (1979) trata a D. parvifolia C.V.Morton (y consecuentemente a D. mortoniana) como sinónimo de D. stenophylla (Donn.Sm.) H.E.Moore. Al revisar el holotipo de D. parvifolia C.V.Morton (P.H. Allen 1949, MO) es evidente que es distinta a $D$. stenophylla. Ambas se diferencian debido a que $D$. stenophylla es usualmente anisofila, con láminas y pedicelos glabros, lóbulos del cáliz oblongos, distintos casi hasta la base y corola oblicua en el cáliz, glabra y no conspicuamente ventricosa hacia la boca. Al contrario, D. mortoniana (basada en $D$. parvifolia C.V.Morton) tiene hojas isofilas a subisofilas en cada par, algunas maduras estrigulosas en el haz, pedicelos glabros a estrigulosos en la base, lóbulos del cáliz ovados e imbricados y corola horizontal en el cáliz, pilosa y conspicuamente ventricosa hacia la boca. El espécimen H. Kennedy et al. 4568 (CR, con un duplicado en MO y otro del cual desconozco su destino), concuerdan satisfactoriamente con el tipo de $D$. parvifolia, razón por la cual $D$. mortoniana es un nuevo registro en la flora de Costa Rica. El espécimen citado había sido anotado como D. aciculata Wiehler, descrita de Panamá, una especie muy distinta y hasta ahora no observada en Costa Rica.

Agradecimientos. Quisiera agradecer a Silvia Troyo por su ilustración y por comentarios al manuscrito, a Frank Almeda por su ayuda en la diagnosis latina y a Nelson Zamora por sus comentarios a la clave. Apoyo económico para el trabajo de campo se obtuvo gracias a la Iniciativa Darwin.

\section{Literatura Citada}

Burtt, B.L. \& Wiehler, H. 1995. Classification of the family Gesneriaeae. Gesneriana 1: 1-4.

Skog, L.E. 1979. Family 175, Gesneriaceae. In: Woodson, R.E \& Schery, R.W. (eds.). Flora of Panama. Part IX. Ann. Missouri Bot. Gard. 65(3): 783-996.

Wiehler, H. 1983. A synopsis of the neotropical Gesneriaceae. Selbyana 6: 1-219.
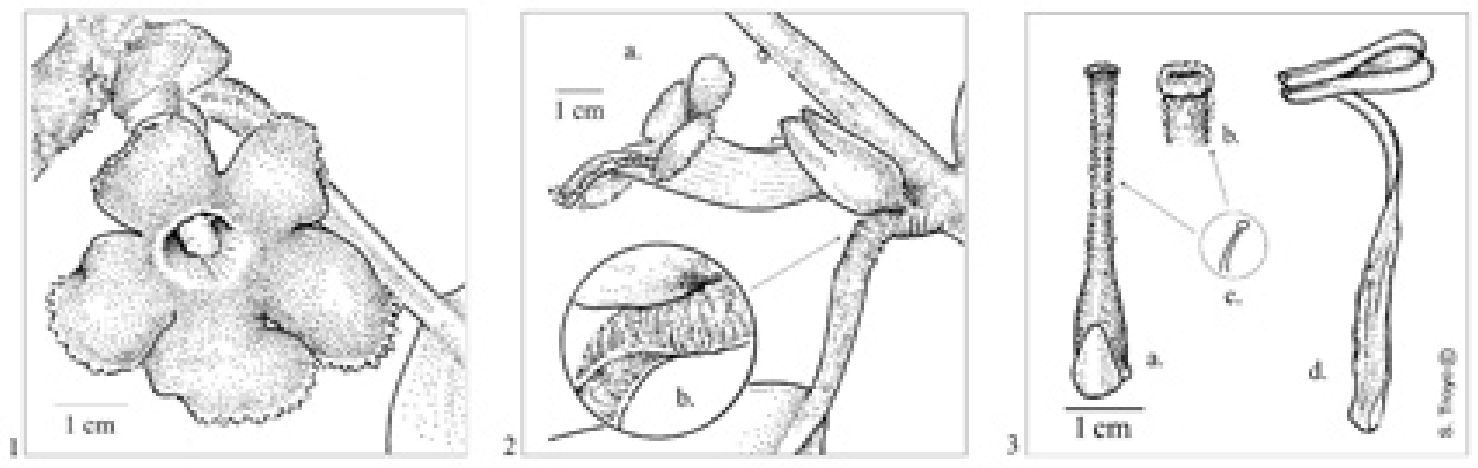

1. Vista frontal de la corola. 2a. Vista lateral de la corola y del cáliz. 2b. Detalle del pecíolo (nótese superficie lepidota). 3a. Vista dorsal de ovario, glándula nectarífera y pistilo. 3b. Estigma. 3c. Detalle del indumento. 3d. Estambre. 

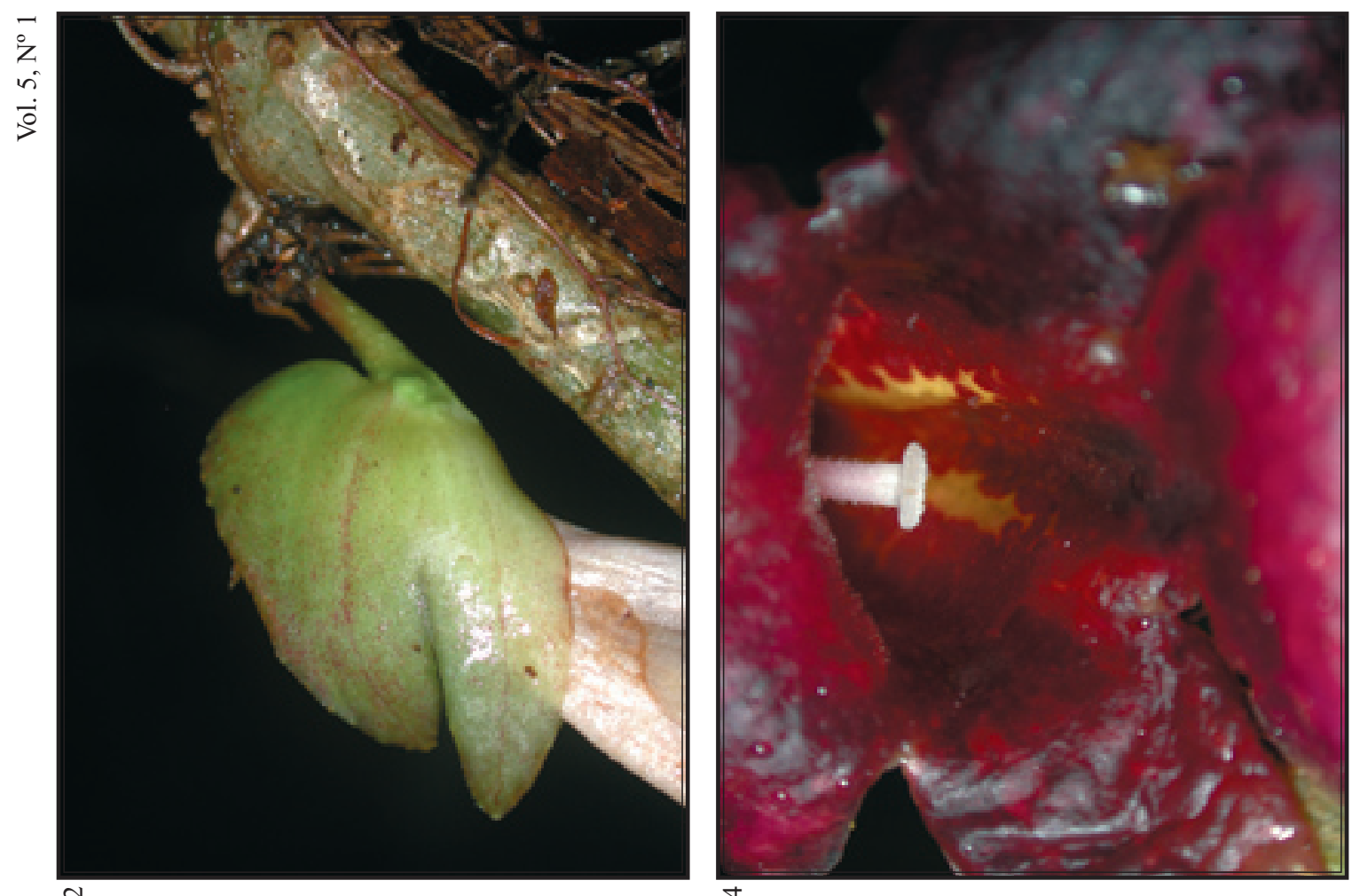

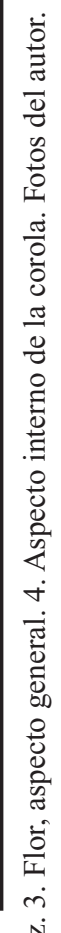
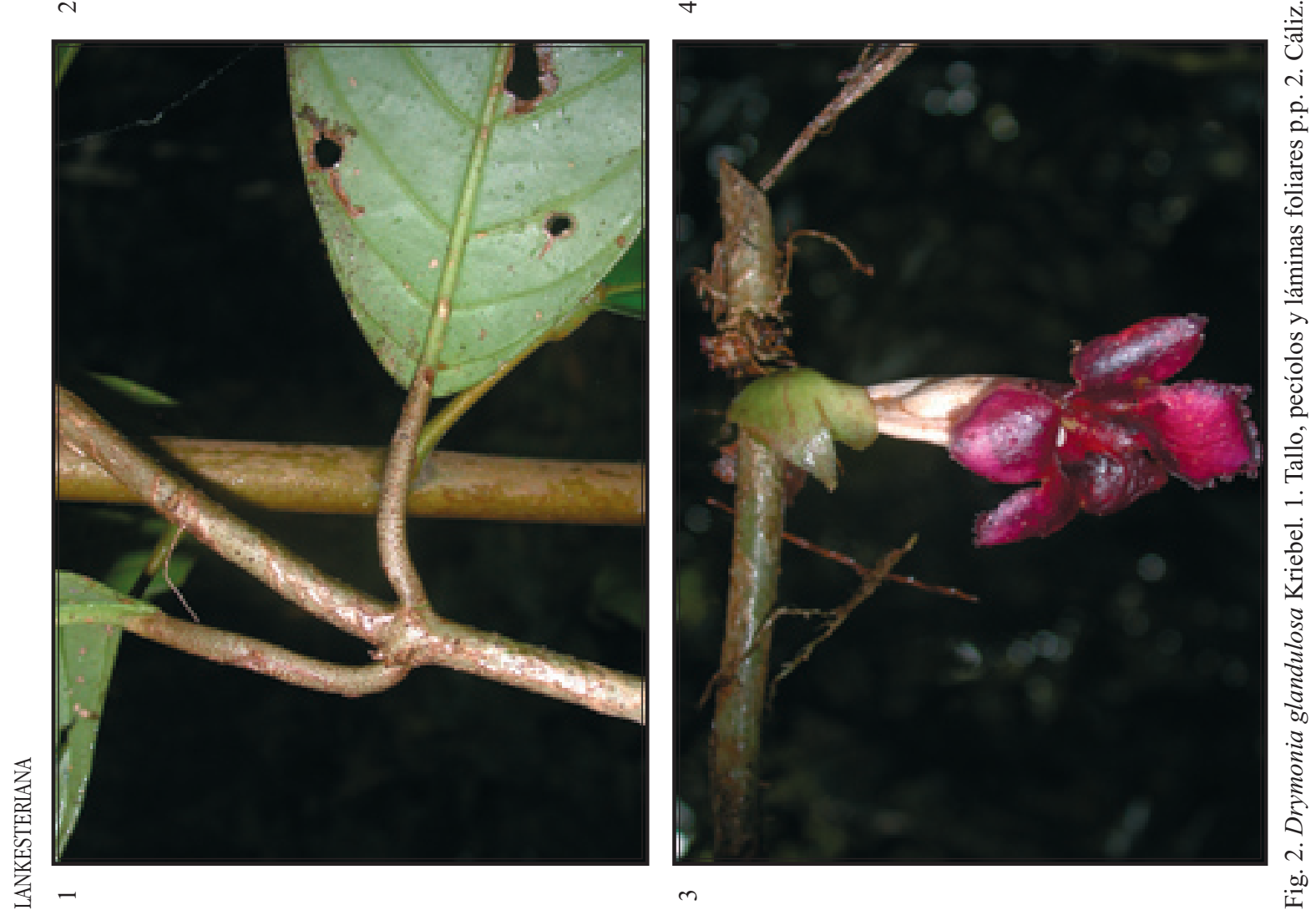